\title{
Imaging polarimetry in the LWIR with microgrid polarimeters
}

\author{
J. S. Tyo \\ College of Optical Sciences, University of Arizona, Tucson, AZ 85721, USA
}

\begin{abstract}
Microgrid polarimeters have emerged over the past decade as a viable tool for performing real-time, highly accurate polarimetric imagery. A microgrid polarimeter operates by integrating a focal plane array (FPA) with an array of micropolarizing optics. Mircrogrids have the advantage of being relatively compact, rugged, and inherently spatiotemporally aligned. However, they have the single disadvantage that the various polarization measurements that go into estimating the Stokes parameters at a particular pixel are actually coming from separate locations in the field. Hence, a microgrid polarimeter performs best where there is no image information, obviating the need for an imaging polarimeter! Recently we have been working with a LWIR microgrid polarimeter at the College of Optical Sciences. Our instrument is a DRS Sensors \& Targeting Systems $640 \times 480 \mathrm{HgCdTe} \mathrm{FPA}$ with linear polarizers at $0^{\circ}, 45^{\circ}, 90^{\circ}$, and $135^{\circ}$ [1]. In this paper we will review our recent results that derive methods for artifact-free reconstruction of band limited imagery.
\end{abstract}

\section{Concept of microgrid polariemter}

Microgrid polarimeters have emerged over the past decade as a viable tool for performing polarimetric imagery. A microgrid polarimeter operates by integrating a focal plane array (FPA) with an array of micropolarizing optics as shown in Fig. 1.

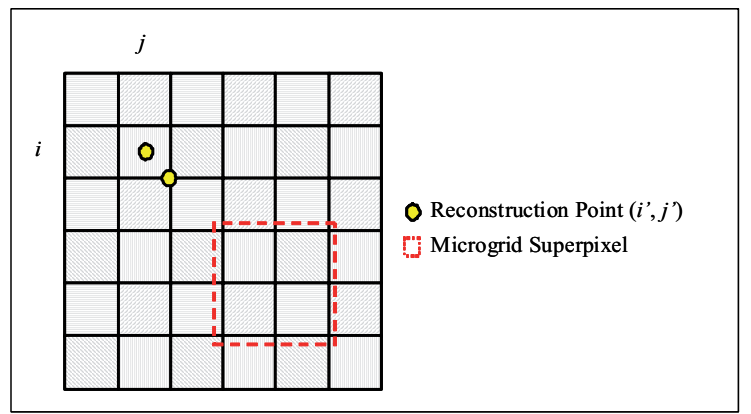

Fig. 1. Layout for the common linear Stokes microgrid polarimeter that measures $\mathrm{s} 0, \mathrm{~s} 1$, and $\mathrm{s} 2$ only.

\section{Image Processing Chain for Microgrid Imagery}

Figure 2 shows the general processing chain that we use for the various stages of Microgrid processing. We have worked on all elements of this chain, but in this paper we concentrate only on the demodulation and estimation blocks.

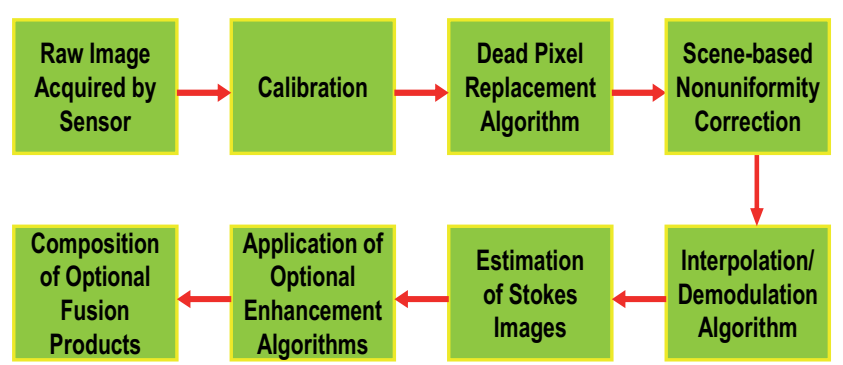

Fig. 2. Image chain sequence for calibration, correction, and processing of microgrid polarimeter data..

When the microgrid array is considered as a spatial modulator, we are able to take the Fourier transform of the modulated intensity as shown in Figure 3.

With knowledge of the information distribution in the spatial frequency plane, it is now possible to define a new band limit criterion for polarization imagery whereby , where $\mathrm{W} 0$ and $\mathrm{W} 1$ are the spatial bandwidths of the s0 and polarization channels, respectively, and the frequency is expressed in normalized units.

This is an Open Access article distributed under the terms of the Creative Commons Attribution-Noncommercial License 3.0, which permits unrestricted use, distribution, and reproduction in any noncommercial medium, provided the original work is properly cited. 


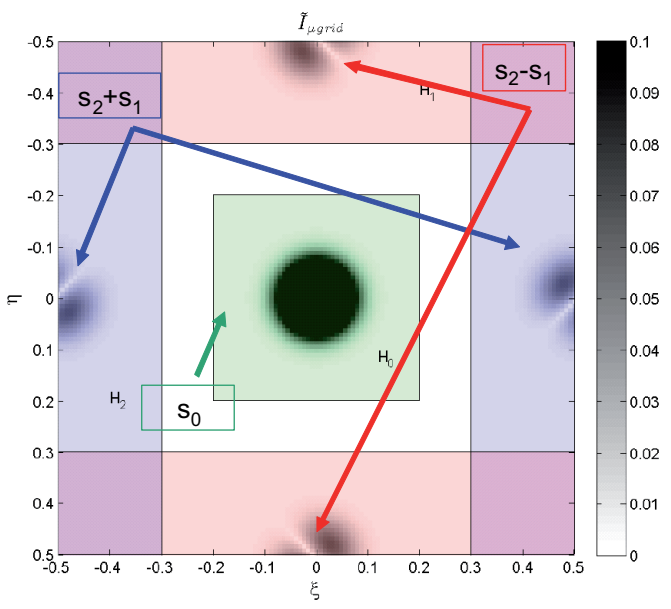

Fig. 3. Fourier transform of modulated intensity in a microgrid polarimeter. The side bands carry the polarization information.

Figure 4 shows and example of this applied to real imagery where polarimetric aliasing is completely eliminated.
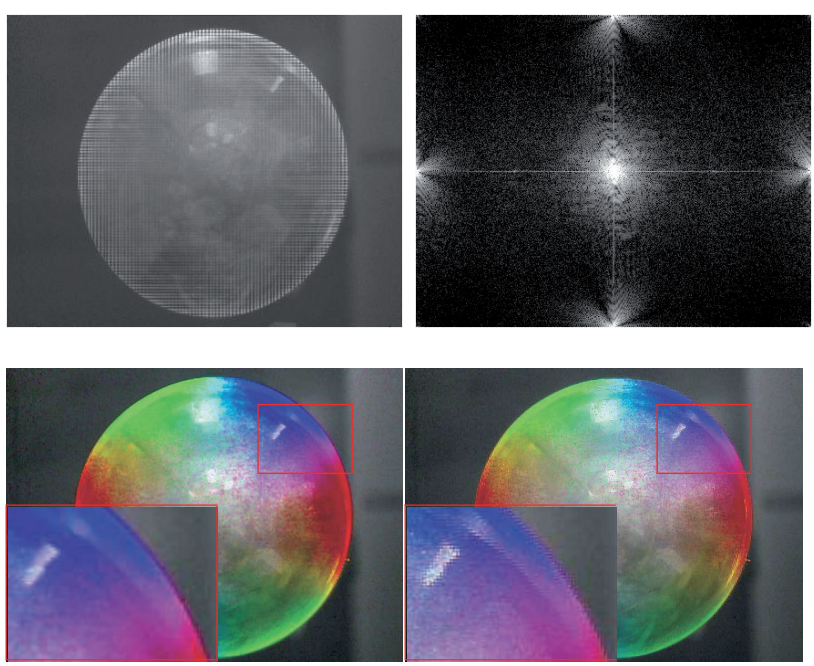

Fig. 4. Band limited polarization reconstruction in the frequency plane eliminates polarimetric aliasing near edges and other high spatial frequency featers.

\section{References}

1. D. L. Bowers, J. K. Boger, L. D. Wellems, S. E. Ortega, M. P. Fetrow, J. E. Hubbs, W. T. Black, B. M. Ratliff, and J. S. Tyo, Opt. Eng., 47, 046403 (2008) 